\title{
Die Bedeutung der pleistozänen Vogelfaunen der Höhlen im Ungarischen Mittelgebirge
}

\author{
Von Tibor FarKas ${ }^{1}$ )
}

Aus den umfassenden Berichten über die bisher bekannten fossilen und subfossilen Vögel von Lambrecht (1933) und Wetmore (1950) geht deutlich hervor, daß ungefähr ein Drittel der in beiden Arbeiten angeführten Formen zum Pleistozän gehört. Bei diesem Sachverhalt kommt den äußerst reichen quaternären Vogelfossilienfunden der verschiedenen Höhlen des Ungarischen Mittelgebirges eine entscheidende Bedeutung zu. Vornehmlich dem aus jenen Höhlen zutage geförderten Material ist es zu verdanken, daß die quaternäre Ornis des Karpatenbeckens heute ziemlich eingehend bekannt ist, was sich immerhin kaum noch über ein anderes Gebiet in der Paläarktis behaupten läßt. Seit Lambrecht noch vor dem ersten Weltkrieg seine ersten diesbezüglichen Arbeiten publizierte, erweckten jene Höhlenfaunen das uneingeschränkte Interesse der ungarischen Paläontologen. So ist es heute schon schwer möglich, ohne Berücksichtigung dieser Höhlenfaunen irgendeine Rekonstruktion von zoogeographischen Verhältnissen in Europa während der quaternären Eiszeit vorzunehmen.

Unterstützt durch die gleiche Auffassung in der Botanik (Soó, 1945; Zólyomi, 1953; Andreanszky, 1954), nehmen heute die meisten ungarischen Zoologen an, daß das Karpatenbecken auch während der maximalen Ausbreitung der Inlandeisdecke im Riß und Würm höchstens ein pseudoperiglaziales Gebiet gewesen sein könnte. Damit lag aber auch die erste Schlußfolgerung auf der Hand; falls diese Annahme richtig ist, so müßte das Karpatenbecken wegen seiner wie hierzu geschaffenen geographisch-klimatischen Lage während der quaternären Eiszeit unter den sich fortwährend ändernden Verhältnissen des Vormarsch-Rückzuges der skandinavischen Eisdecke eine ziemlich ausgeprägte Rolle des Faunenrefugiums gespielt haben. - Obwohl sich diese Annahme in Mitteleuropa auch allgemeiner Beliebtheit erfreut und obwohl zahlreiche paläontologische und paläobotanische Funde diese auch zu bestätigen scheinen, darf man jedoch nicht übersehen, daß diese Refugiumtheorie vorerst nur in bezug auf die Interstadiale

1) Barberspan Bird Research Station, Republic of South Africa. 
genügende Beweise vorbringen kann. Die Funde aus den Stadialen sind heute nur noch spärlich, und somit bleibt die unanfechtbare Bestätigung dieser Theorie vorläufig noch der Zukunft vorbehalten. Immerhin, die bis jetzt bekanntgewordenen Höhlenfaunen scheinen des öfteren auf etliche, ein mild-gemäßigtes Klima vorziehende Arten aufweisender Faunen auch während der Interstadialen hinzudeuten. Diese Behauptung wird wahrscheinlich in der näheren Zukunft durch bisher unbearbeitete reichhaltige Höhlenfunde noch mehr verstärkt (briefliche Mitteilung von Jánosy, 1962).

Man darf aber auch den Begriff Refugium nicht allzu eng auffassen; zu Zeiten, als in Westeuropa das eisfreie Gebiet zwischen der alpinen und skandinavischen Eisdecke zu schmal geworden war, mußte ein Großteil der dortigen Populationen zu den nächstliegenden, für die betroffenen Arten immerhin noch erträgliche Bedingungen bietenden Gebieten wandern. Daß auch für diese Arten das Karpatenbecken ein Refugium darstellte, geht aus manchen, einen starken glazialen Charakter aufweisenden stadialen Höhlenfunden hervor. Mit diesem Hinweis auf seine doppelte Refugiumrolle wird die Bedeutung des Karpatenbeckens noch weiter erhöht. Die günstige geographische Lage im Nordwesten durch hohe, aber keine zusammenhängende Eisdecke tragende Gebirgsketten abgeschirmt, im Südwesten ziemlich offen und nicht zuletzt die sich aus dieser speziellen Lage vermutlich ergebenden örtlichen oder mikroklimatischen Begebenheiten, je nach Höhenlage und Entfernung vom Inlandeis und von der mediterranen Westwindzone, verstärken die Annahme von sehr mannigfaltigen und wechselhaften Verhältnissen in diesem Gebiet während der letzten zwei Glazialen. Hierbei waren bald die glazialen, bald die gemäßigten Faunentypen überwiegend, wahrscheinlich ohne daß der eine oder andere Typ gleichzeitig aus dem ganzen Gebiet verdrängt worden wäre. Für beide Faunentypen war aber sicherlich das Karpatenbecken jeweils das Randgebiet, wo sie sich noch mehr oder weniger halten konnten und von wo aus die vom Eis freigewordenen Gebiete wieder besiedelt werden konnten.

Zum Abschluß dieser einleitenden Überlegungen sollte noch erwähnt werden, daß die ungarischen Paläontologie außer der Paläobotanik auch eine weitere Hilfe von der geologischen Eiszeitforschung zum Aufbau und zur Unterstützung der Refugiumtheorie erhielt. Nachdem Milankovic (1920) die Forschung der Ursachen und der Periodizität der quaternären Eiszeit durch seine hypothesenfreie Theorie auf eine exakte astronomische Grundlage gesetzt hatte, wurde diese Theorie vornehmlich wegen scheinbarer Berechnungsfehler teilweise stark kritisiert (Penck, 1938), anderenteils aber, wenn auch indirekt, be- 
stätigt (Soergel, 1937). Erst Bacsák (1954) gelang es durch eine Reihe von diesbezüglichen Veröffentlichungen, die anfänglichen Mängel von Milankovics Theorie zu beseitigen und diese - wenigstens in Mitteleuropa - zu allgemeiner Anerkennung zu verhelfen. Abgesehen von der astronomisch-mathematischen Genauigkeit der Milankovic-Soergel-Bacsákschen Theorie, ermöglichte diese - im Gegensatz zu Pencks vornehmlich auf die alpinen Vereisungen gegründeten Auffassungen eine viel subtilere Gliederung der vier quaternären Vereisungen. Die sich aus dieser Möglichkeit ergebenden Perspektiven wurden alsbald erkannt, und schon zu gleicher Zeit mit der hier angeführten Debattenschrift von Bacsák publizierte Kriván (1954) eine Arbeit, in der die Dynamik der einander fortwährend abwechselnden vier solaren Klimatypen von Bacsák mit einer neuen, sinnvollen Nomenklatur belegt worden ist. Jedoch, um eine Verwirrung zu vermeiden, wird hier von den schon eingebürgerten Penck-Eberlschen Begriffen Glazial - Interglazial und Stadial - Interstadial Gebrauch gemacht, um so mehr als auch die Zeitangaben der verschiedenen Forscher, die hier angeführt werden, nach dieser Nomenklatur gemacht worden sind. Diesem Prinzip entsprechend werden hier auch sämtliche Arten mit ihren von den betreffenden Autoren verwendeten Synonymen bezeichnet, ohne Rücksicht auf die eben gültige zoologische Benennung.

Als erste quaternäre Glazialperiode, welche auf das Karpatenbecken eine entscheidende Wirkung ausgeübt hatte, ist zweifellos der Riß anzuerkennen. Jedoch sind vorläufig weder aus dieser Zeit noch aus dem Riß-Würm-Interglazial Vogelfossilien aus dem Ungarischen Mittelgebirge bekannt. Bloß aus dem allgemeinen Faunen- und Vegetationsbild der erwähnten Perioden kann man gewisse Rückschlüsse auf die synchrone Vogelfauna ziehen. Die in den fossilen Faunen des Riß mit einem starken prozentualen Anteil vertretenden Höhlentiere vom glazialen Typ (Höhlenbär und Löwe) bleiben auch den späteren RißWürm-Faunen erhalten, wobei aber in diesem Interglazial - als letztes Aufgebot - noch einmal südliche Formen mit auftreten (Felis pardus, Hippopotamus, Hystrix, Testudo usw.). In den Wäldern des Karpatenbeckens im Riß-Würm herrschten besonders Quercus, Corylus, Junglans und Thuja (Mottl, 1953). Daher gehören die ersten glazialen Vogelfunde aus Ungarn ins Würm I; sie sind aber vorerst nur noch die bescheidenen Vorboten der späteren reichen Höhlenfaunen des Beckens. Aus Tata (mittlerer Abschnitt des Ung. Mittelgeb.) wurden von Lambrecht (1933) Fossilien von Circus cyaneus, Numenius arquatus und Lyrurus tetrix beschrieben.

Die bisher älteste bedeutendere Höhlenfauna des Gebietes gehört dem Würm-I-II-Interstadial an. Aus den unteren braunen Lehm- 
schichten der Istállóskö-Höhle (NW des Ung. Mittelgeb.) kamen die folgenden Arten zum Vorschein (Jánosy, 1951): Corous corone, Coloeus monedula, Pica pica, Nucifraga caryocatactes, Pyrrhocorax graculus, Loxia curvirostra, Alauda gen., Turus viscivorus, Hirundo rustica, A pus apus, Dendrocopus maior, Nyctea sive Bubo sp., Asio flammeus, Aegolius funereus, Surnia ulula, Falco subbuteo sive columbarius, Falco vespertinus, Falco tinnunculus, Anas platyrhynchos, Charadrius gen. Porzana porzana, Crex crex, Tetrao urogallus, Lyrurus tetrix, Lagopus lagopus und Lagopus mutus. Ferner sind hier noch als unsicher bestimmbare Fossilien unter Fragezeichen Sturnus sp., Coccothraustes coccothraustes und Pinicola enucleator angeführt.

Aus denselben braunen Lehmschichten dieser Höhle brachten die Ausgrabungen auch eine Feuerstätte des Hochaurignac-Menschen zutage. Einige Holzkohlenreste, die hier vorgefunden waren, wurden durch Stieber (1952) anthrakotomisch als Pinus montana, Pinus cembra und Pinus sylvestris bestimmt, worauf Stieber sofort auf ein kälteres Klima schloß. - Es ist auch nicht zu übersehen, daß die relativ starke glaziale Einfärbung der Ornis jener braunen Lehmschichten in der Höhle weitgehend die Auffassung bezüglich eines kälteren Klimas zu unterstützen scheint. - Nun sind aber in derselben Vogelfauna auch solche Arten vertreten, welche nur mit größtem Vorbehalt als typische Taiga-Bewohner zu bewerten sind. Auch bezüglich der Holzkohlenreste muß man sehr vorsichtig sein, denn die heutige untere Klimaxgrenze für Pinus sylvestris liegt bei etwa $450 \mathrm{~m}$ ü.d.M. im südlichen Ungarischen Mittelgebirge und für Picea excelsa bei $650 \mathrm{~m}$ ü.d.M. (Soó, 1945). Die Istállóskö-Höhle liegt aber in einer Höhe von etwa $700 \mathrm{~m}$ ü.d.M. und ist auch heute noch mit einem Nadel-Laub-Mischwald umgeben. - Daß Nadelbäume nicht unbedingt ein rauhes Klima voraussetzen, kann hier auch nur zusätzlich erwähnt werden.

Genau wie die Vogelreste durch eine selektive Tätigkeit - wahrscheinlich vor allem von Eulen - in die Höhlen kamen, verdanken wir die erwähnten Holzkohlenreste einer noch selektiveren Tätigkeit des Aurignac-Menschen, der sich vermutlich - wie alle Primitiven - die nützlichen und angenehmen Wirkungen des Lagerfeuers durch die geringste Kraftanwendung zu verschaffen suchte. Der Umstand, daß diese Höhle auf einem Steilhang liegt, macht es wahrscheinlich, daß der Aurignac-Mensch Feuerholz aus den nächstliegenden Beständen, also aus der unmittelbaren Nähe der Höhle, holte. Wenn dort in der Höhe des Berges im Würm I-II eventuell auch Laubgewächse vorhanden waren, könnte man doch als fast sicher annehmen, daß der Mensch aus praktischen Gründen den Vorzug unbedingt den leichter brennenden harzigen Nadelhölzern gegeben hat. - Diese Überlegung 
dürfte auch zu Vorsicht mahnen, ehe man an Hand solcher spärlichen Funde ohne Berücksichtigung der Umstände unbedingt auf ein rauheres Klima schließt.

Des weiteren ist durch die Tatsache, daß die mitteleuropäische Vereisungskurve (Krivan, 1954) im Würm I-II negativ ist, noch Vorsicht geboten. Daher dürfte man unter Berücksichtigung aller hier angeführten Indizien das Klima des Karpatenbeckens höchstens mit dem des heutigen Baltikum gleichsetzen.

Die nächstfolgende Periode Würm II ist, was Vogelfossilien anbelangt, vorläufig steril; somit ist aber diese Periode, die zugleich den Höhepunkt der quaternären Eiszeit in Europa darstellt, die Achillesferse der erwähnten Refugiumtheorie, zumindest wenn man sie mit Bezug auf die nichtglazialen Arten gelten läßt. Deshalb widmen auch die meisten Gegner der Refugiumhypothese ihr vornehmliches Interesse dem Würm II. Um hier nur auf eine der letzten diesbezüglichen Auffassungen einzugehen, muß man auch einige leicht angreifbare Punkte von Moreau (1955) verweisen. Seine ,,Bird Geography“ Europas im Würm II ist fast ausschließlich auf die paläobotanischen Vegetationskarten von Büdel, Frenzel und Troll gegründet, wobei er bemerkt: ,...the available fossils are of comparative little help, mainly because so few of the deposits containing them can be dated with the necessary stratigraphic exactness." Es erübrigt sich hier, darauf hinzuweisen, daß weder Paläontologie noch Paläobotanik je über eine ,,bessere" und ,,exaktere" stratigraphische Bestimmungsmethode als die andere Disziplin verfügte; also man geneigt ist, die paläobotanischen stratigraphischen Bestimmungen als richtig anzuerkennen, muß man konsequenterweise diese Gunst auch der Paläontologie erweisen.Abgesehen aber von einer solchen unbegründeten Bevorzugung der Verläßlichkeit von stratigraphischer Exaktheit der einen oder anderen Disziplin der Paläobiologie, wird weiter unten an Hand einiger paläobotanischen Funde veranschaulicht, welche Unstimmigkeiten auch bei der Rekonstruktion des Vegetationsbildes durch voreilige oder nicht genug umsichtige Deutung solcher Funde entstehen können.

Eine weitere Hypothese von Moreau, die man auch mit besonderer Vorsicht aufzunehmen hat, lautet: ,In conclusion it may be noted that the vegetational picture of Europe at the height of the glaciation does not accord with the view that there were two 'refuges,' a southeastern and southwestern." Da Würm III im Karpatenbecken mit ziemlich heterogenen Faunen reichlich belegt ist, würde an sich der Gedanke naheliegen, aus verschiedenen Gründen wenigstens annähernd ähnliche Verhältnisse auch dem Würm II zuzueignen. Jedoch muß man wieder einmal betonen, daß ausreichende Beweise bezüglich des 
Charakters des mitteleuropäischen Würm II vorläufig noch nicht vorhanden sind, daher müssen vorerst ,,optimistische" Ableitungen, wie im vorigen Satz erwähnt, genauso wie allzu ,,pessimistische“ Bewertungen des Glazials Würm II nur als auch mögliche Alternativen behandelt werden.

Die Reihe der Vogelfossilien fängt erst wieder mit dem Würm II-III (protosolutréen) an. Am ausgiebigsten ist diese Ornis aus der OttoHerman-Höhle (NO des Ung. Mittelgeb.) belegt. Lambrecht (1915) hatte hier die folgenden Arten nachgewiesen: Anas platyrhynchos, Lyrurus tetrix, Lagopus lagopus, Lagopus mutus, Tringa totanus, Aegolius funereus, Bubo bubo, Asio flammeus, Dendrocopus major, Turdus viscivorus, Turdus ericetorum, Cinclus cinclus, Sturnus vulgaris, Pyrrhula pyrrhula und Coccothrausthes coccothrausthes. - Dazu kam noch aus der Szeleta-Höhle (NO des Ung. Mittelgeb.) Gypaetus barbatus (Lambrecht, 1933).

Als aufschlußreiche Antithese zu dieser - immerhin weniger intensiv als im Würm I-II - auch glazial eingefärbten Ornis dienen einige paläobotanische Funde aus dem Solutréen zweier Höhlen: aus der Szelim-Höhle (mittlerer Abschnitt des Ung. Mittelgeb.) bestimmten anthrakotomisch Sárkány \& Stieber (1950) Fraxinus (excelsior?) und Tilia(platyphyllos?) und aus der Szeleta-Höhle (NO-Abschnitt des Ung. Mittelgeb.) Stieber (1952) wiederum nur Laubbäume (Quercus, Tilia, Acer und Crategus). Beide Autoren erwähnen, daß bis dahin aus dem Solutréen Ungarns nur Nadelbäume (Pinus cembra, Larix sp. und auch eventuell Pinus montana) bekannt waren. Sichtlich aus diesem Grunde sahen sie sich veranlaßt anzunehmen, daß das Klima jener Zeit bedeutenden Schwankungen ausgesetzt war. Daß bei dieser Stellungnahme - ähnlich der Beurteilung der Holzkohlenreste aus der Istállóskö-Höhle - die erwähnten Autoren die während der Eiszeit besonders wichtige Rolle der vertikalen Verbreitungsgrenzen außer acht ließen und statt dessen zu der weniger plausiblen Hypothese von jähen Klimaschwankungen griffen, beweist die Unhaltbarkeit der Methode, aus wenigen und zufälligen Funden gleich weittragende Diagnosen aufzustellen. - Da die mitteleuropäische Vereisungskurve im Würm II-III schwach positiv ist, hätte man Laubbäume eher aus diesem Interstadial als aus Würm I-II erwarten können. Jedoch, wie wir eben gesehen haben, führte der Zufall gerade zum Entgegengesetzten, ein Umstand, welcher zugleich auf in Zukunft zu erwartende Überraschungen auf dem Gebiet diesbezüglicher Forschung hindeutet.

Im nun zu behandelnden Würm III war der Wirkungsgrad der Vereisungskurve beinahe von derselben Intensität wie im Würm II; jedoch war dieser Stadial - vorläufig der letzte - von verhältnismäßig 
kurzer Dauer. Immerhin, aus allen Phasen dieses Stadials stehen uns die bisher reichhaltigsten Vogelfossilienfunde zur Verfügung. Kretzoi (1952) beschrieb aus der Csákvárer-Höhle (südlicher Abschnitt des Ung. Mittelgeb.) einige Arten vom glazialen Typ und ordnete sie ins untere Würm III ein: Tetrao urogallus, Lagopus lagopus, Lagopus mutus, Anas platyrhynchos, Aythya nyroca, Coloeus monedula.

Aus den mittleren Zeiten des Stadials vorliegende Höhlenfaunen tragen alle einen starken glazialen Charakter, wozu auch der Umstand beiträgt, daß die prozentuale Beteiligung der glazialen Leitarten (z.B. Lagopus lagopus, Lagopus mutus, Anas platyrhynchos, Tetrao urogallus) an diesen Faunen besonders hoch ist. Jedoch unausbleiblich werden überall auch Arten repräsentiert, welche man nicht als ausschließliche Taiga-Bewohnerinnen vorstellen kann. - Im Bükk-Gebirge (NO des Ung. Mittelgeb.) unweit der schon öfters erwähnten Istállóskö-Höhle liegt in einer Höhe von etwa $850 \mathrm{~m}$ die Peskö-Höhle, welche uns auch eine Würm-III-Ornis lieferte (Lambrecht, 1912): Lyrurus tetrix, Tetrao urogallus, Tetrao medius, Lagopus lagopus, Lagopus mutus, Asio flammeus, Picus canus, Turdus pilaris, Coloeus monedula, Pica pica, Nucifraga caı yocatactes, Pyrrhocorax pyrrhocorax.Weitere Angaben zur Ornis des Würm III lieferte die ebenfalls im Bükk-Gebirge liegende Balla-Höhle. Obwohl hier die Fossilien aus drei übereinandergelagerten Schichten hervorkamen, bleibt das Gepräge der Funde stark glazial, und Lambrecht (1912) ordnete sie in die mittleren Zeiten des Stadials ein: Mergus merganser, Anas platyrhynchos, Branta ruficollis, Lyrurus tetrix, Tetrao urogallus, Lagopus lagopus, Lagopus mutus, Crex crex, Larus ridibundus, Aegolius funereus, Surnia ulula, Asio flammeus, Picus canus, Turdus ericetorum, Turdus pilaris, Nucifraga caryocatactes, Pyrrhocorax pyrrhocorax, Pyrrhula pyrrhula.Die beinahe gleiche artliche Zusammensetzung der Ornis aus der Öregkö-Höhle bei Bajót (mittlerer Abschnitt des Ung. Mittelgeb.) verrät uns den synchronen Charakter beider letzten Faunen: Mergus albellus, Anas platyrhynchos, Circus cyaneus, Lyrurus tetrix, Lagopus lagopus, Lagopus mutus, Perdix perdix, Rallus aquaticus, Columba palumbus, Asio flammeus, Turdus ericetorum, Turdus pilaris, Corvus corax, Pica, Nucifraga caryocatactes, Pyrrhocorax pyrrhocorax.

Den reichsten aller ungarischen Höhlenfunde aus dem Würm III stellen die Fossilien aus der Felsnische von Pilisszántó (W von Budapest) dar. Die heterogene Zusammensetzung dieser Fauna, wo neben den an Individuenzahl immer noch überwiegenden glazialen Arten auch schon zahlreiche südliche Formen repräsentiert sind, bezeichneten Lambrecht und Kormos (1914) als Blütezeit dieser Ornis die Endphase des Würm III (Magdalénien): Podiceps auritus, Mergus sp., 
Anser fabalis, Anser sp., Anas platyrhynchos, Anas strepera, Anas querquedula, Aythya nyroca, Buteo rufinus, Circus cyaneus, Circus sp., Falco culumbarius, Falco cherrug, Falco tinnunculus, Falco vespertinus, Numida meleagris, Coturnix coturnix, Perdix perdix, Lyrurus tetrix, Lagopus lagopus, Lagopus mutus, Columba oenas, Cuculus canorus, Athene noctua, Glaucidium passerinum, Surnia ulula, Nyctea scandiaca, Asio otus, Asio flammeus, Dendrocopus major, Picus canus, Hirundo rustica, Acrocephalus arundinaceus, Turdus viscivorus, Turdus ericetorum, Turdus pilaris, Turdus sp., Lanius minor, Lanius senator, Corous corax, Corsus frugilegus, Coloeus monedula, Rallus aquaticus, Crex crex, Porzana porzana, Himantopus himantopus, Vanellus vanellus, Tringa sp., Philomachus pugnax, Calidris alpina, Capella media, Scolopax rusticola, Numenius sp., Larus ridibundus, Sterna hirundo, Syrrhaptes paradoxus, Columba palumbus, Pica pica, Nucifraga caryocatactes, Garrulus glandarius, Pyrrhocorax pyrrhocorax, Oriolus oriolus, Sturnus roseus, Sturnus vulgaris, Emberiza calandra, Fringilla coelebs, Loxia curvirostra, Pinicola enucleator, Pyrrhula pyrrhula, Coccothraustes coccothraustes, Motacilla alba, Galerida cristata.

Mit diesen bis jetzt reichsten Höhlenfunden ist aber die Reihe noch keineswegs abgeschlossen. Aus den oberen Lehmschichten der schon erwähnten Csákvárer Höhle bestimmte Kretzoi (1952) noch weitere Arten, wobei er bemerkte, daß über zweihundert weitere Arten in diesem Funde vorläufig noch nicht bearbeitet werden konnten: Anatide, Anas platyrhynchos, Anas crecca, Gallus domesticus, Phasianus sp., Perdix perdix, Scolopax rusticola, Aquila chrysaëtos, Bubo bubo, Nyctea scandiaca, Coloeus monedula, Chelidon sp.

Diese letzten Höhlenfunde führen schon aus dem Würm III ins postglaziale Spätmagdalénien hinüber, dessen Ornis auch durch eine Serie von Höhlenfaunen musterhaft belegt ist. Die zunehmende Milderung des Klimas im späten Würm III läßt sich durch die Abnahme der Individuenzahl der glazialen Leitarten bei gleichzeitiger Zunahme der Individuen und Arten der nïchtglazialen Elemente deutlich erkennen. Die oberen Schichten der Remete-Höhle (W bei Budapest) wurden schon durch Kormos (1914) erschlossen, wobei die Bestimmung die folgende Faunenliste ergab: Anas platyrhynchos, Anas penelope, Anas crecca, Aythya nyroca, Anser albifrons, Buteo buteo, Buteo lagopus, Accipiter nisus, Accipiter gentilis, Circus cyaneus, Falco columbarius, Falco tinnunculus, Falco vespertinus, Numida meleagris, Coturnix coturnix, Lagopus lagopus, Lagopus mutus, Lyrurus tetrix, Tetrao urogallus, Rallus aquaticus, Crex crex, Porzana porzana, Columba palumbus, Aegolius funereus, Surnia ulula, Strix uralensis, Nyctea scandiaca, Asio otus, Asio flammeus, Dendrocopos major, Hirundo rustica, Turdus 
viscivorus, Turdus ericetorum, Turdus pilaris, Lanius collurio, Corsus corax, Corsus cornix, Pica pica, Nucifraga caryocatatctes, Garrulus glandarius, Pyrrhocorax pyrrhocorax, Oriolus oriolus, Plectrophenax nivalis, Loxia curvirostra, Phyrrula pyrrhula.

Mit einer sehr ähnlichen artlichen Zusammensetzung lieferte die Puskaporos-Höhle (Bükk-Gebirge) auch eine reiche EndmagdalénienOrnis: Ardea cinerea, Mergus albellus, Anas platyrhynchos, Anas penelope, Anas crecca, Aythya ferina, Aythya nyroca, Haliaëtus albicilla, Buteo lagopus, Falco peregrinus, Falco columbarius, Falco vespertinus, Coturnix coturnix, Caccabis saxatilis, Lyrurus tetrix, Tetrao urogallus, Lagopus lagopus, Lagopus mutus, Rallus aquaticus, Crex crex, Porzana porzana, Vanellus vanellus, Capella media, Scolopax rusticola, Limosa Timosa, Numenius sp., Larus ridibundus, Aegolius funereus, Surnia ulula, Nyctea scandiaca, Asio flammeus, A pus apus, Dendrocopos major, Turdus viscivorus, Turdus pilaris, Turdus sp., Lanius senator, Corvus corax, Coloeus monedula, Pica pica, Nucifraga caryocatactes, Garrulus glandarius, Pyrrhocorax pyrrhocorax, Sturnus vulgaris, Plectrophenax nivalis, Loxia curvirostra, Emberiza schoeniclus, Parus major, Parus palustris, Anthus trivialis, Galerida cristata. -

\section{ZUSAMMENFASSUNG}

In der vorliegenden Arbeit werden die fossilen Vogelfaunen der Höhlen im Ungarischen Mittelgebirge auf die Rolle hin untersucht, welche sie bei der Unterstützung der Hypothese über den Karpatenbecken als faunistisches Refugium während der letzten quaternären Vereisung spielen können.

Zur Einleitung werden die Beweggründe zur Refugiumhypothese einschließlich die paläobotanischen und glazialtheoretischen Aspekte kurz erörtert.

Da die ersten Vogelfossilien der hier zu behandelnden Höhlenfaunen in den Würm I-II gehören, werden die faunistischen Verhältnisse während des Riß-Glazials nur kurz umrissen. - Des weiteren läßt sich feststellen, daß sämtliche bis jetzt bekannte Faunen bis zum Würm III nur Interstadialfaunen sind, welcher Umstand vorläufig nur eine indirekte Unterstützung für die Refugiumhypothese liefern könnte. Paläobotanische Funde sowie daraus abgeleitete Erwägungen, welche sowohl gegen als auch für die Hypothese sprechen, werden in diesem Abschnitt auch miterörtert.

Zum Abschluß werden die reichhaltigen Höhlenfaunen aus allen Phasen des Würm III als vorläufig eines der stärksten Argumente für die Refugiumhypothese angeführt. Die heterogene Zusammensetzung dieser Faunen läßt gewisse Rückschlüsse auf die Faunen des Würm I und Würm II zu, welche immerhin erst durch zukünftiges Beweismaterial bekräftigt werden müssen. 


\section{SUMMARY}

In the present study, the fossil bird fauna of the caves of the Hungarian Middle Mountains is examined for evidence in support of the hypothesis that the Carpathian Basin may have served as a faunal refugium during the last Quaternary glacial period. As an introduction, the reasons for the refugium hypothesis, including paleobotanical and glacial theoretical aspects, are discussed.

Since the first bird fossils of the cave fauna considered in this paper belong to the Würm I-II, the faunistic conditions of the Riss glacial period are not discussed in detail, The known faunas up to the Würm II are interstadial, which seems to serve only as indirect support for the refugium hypothesis. Paleobotanical evidence, both for and against the hypothesis, is also considered.

In conclusion, the abundant cave faunas of all phases of the Würm III are cited as being - at least at the present time - the most convincing argument for the refugium hypothesis. The heterogeneous composition of these faunas permits certain tentative conclusions regarding the faunas of Würm I and II.

\section{LITERATUR}

Andreánszky, G. - 1954: Paläobotanik. Budapest.

BAcś́ K, Gy - 1954: Pliozän- und Pleistozänzeitalter im Licht der Himmelsmechanik. Acta Geol. Acad. Sci. Hung. $3(4)$ : 305-343.

Jánosy, D. - 1951: Fossile Ornis aus der Höhle von Istállóskö. Aquila. $55-58: 205-223$.

- 1953: Die Fauna der Lambrecht-K.-Höhle. Archeol. Értesitö (Archeol. Ber.) $80(1): 27-28$.

Kadic, O., und Mottr, M. - 1944: Die Höhlen des nordwestlichen BükkGebirges. Barlangkutatás (Höhlenforschung) 17: 85-111.

Kовмоs, T., und Laмввеснт, K. - 1914: Die pleistozäne und prähistorische Vogelfauna der Felsnische am Remetehegy. Mitt. Jahrb. kgl. Ung. Geol. Reichsanst. 22: 391-404.

Kretzor, M. - 1952: Schlußmeldung über die paläontologische Erschließung der Höhle von Csákvár. Jahresber. Geol. Anstalt.: 37-69.

Kriván, P. - 1954: Die klimatische Gliederung des mitteleuropäischen Pleistozäns. Acta Geol. Acad. Sci. Hung. 3 (4) : 357-382.

La mв веснт, K. - 1912: Die fossilen Vögel Ungarns. Aquila 19: 288-320.

— 1915: Die Vögel der Felsnische von Pilisszántó usw. Mitt. Jahrb. kgl. Ung. Geol. Reichsanst. 23: 477-517.

- 1933: Handbuch der Paläornithologie. Berlin.

Milan k ovic, M. - 1920: Théorie mathématique des phénomènes thermiques produits par la radiation solaire. Paris.

Moreau, R. E. - 1955: The bird-geography of Europe in the last glaciation. Acta XI. Congr. Int. Orn. Basel 1954 (Exp. Suppl.).

Мотть, M. - 1941: Interglazialen und Interstadialen im Spiegel der ungarischen Säugetierfauna. Jahresber. kgl. ung. Geol. Anst. Anh. 1941.

PencK, A. - 1938: Die Strahlungstheorie und die geologische Zeitrechnung. Zeitschr. Ges. Erdk. Berlin (9-10). 
SÁrkány, S. ,und Stieber, J. - 1950: Anthrakotom. Untersuchung neueren Holzkohlenfunde aus der Szelim-Höhle. Jahrb. Biol. Inst. Univ. Budapest. (1): 32-37.

Soó, R. - 1945: Növényföldrajz (Pflanzengeographie). Budapest.

Soergel, W. - 1937: Die Vereisungskurve. Berlin.

Stieber, J. - 1952: Anthrakotomitschesskije i xilotomitschesskije issledovanija. Ann. Hist. Nat. Mus. Nat. Hung. 2: 261-280.

Wetmore, A. - 1950: Recent additions to our knowledge of prehistoric birds. Proc. Xth Int. Cong. Orn. Uppsala. 51-74.

Zoly омі, B. - 1953: Die Entwicklungsgeschichte der Vegetation Ungarns seit der letzten Interglazial. Acta Biol. Acad. Sci. Hung. 4 (3-4). 